\title{
Spectroscopic EUV observations of impulsive solar energetic particle event sources
}

\author{
R. Bučík ${ }^{1,2}$, A. Fludra ${ }^{3}$, R. Gómez-Herrero ${ }^{4}$, D. E. Innes ${ }^{2}$, B. Kellett ${ }^{3}$, R. Kumar ${ }^{5}$, and Š. Mackovjak ${ }^{6}$ \\ 1 Institut für Astrophysik, Georg-August-Universität Göttingen, D-37077 Göttingen, Germany \\ 2 Max-Planck-Institut für Sonnensystemforschung, D-37077 Göttingen, Germany, e-mail: bucik@mps .mpg. de \\ 3 STFC Rutherford Appleton Laboratory, Didcot, OX11 0QX, UK \\ 4 Space Research Group, University of Alcalá, E-28871 Alcalá de Henares, Spain \\ 5 Department of Astrophysical Sciences, Princeton University, Princeton, NJ 08544, USA \\ ${ }^{6}$ Institute of Experimental Physics, Slovak Academy of Sciences, Watsonova 47, Košice, Slovakia \\ Received ; accepted
}

\section{ABSTRACT}

\begin{abstract}
Context. The remote observations of solar flare ion acceleration are rather limited. There are theoretical predictions for signatures of ion acceleration in EUV line profiles. Previous tests involve observations of flares with no evidence for energetic ions.

Aims. We aim to examine a source flare of impulsive (or ${ }^{3} \mathrm{He}$-rich) solar energetic particle events with EUV line spectroscopy.

Methods. We inspect all (90+) reported ${ }^{3} \mathrm{He}$-rich flares of previous solar cycle 23 and find only four (recurrent) jets in the field of view of SOHO CDS. The jet with the most suitable spatial and temporal coverage is analyzed in detail.

Results. Two enhanced (non-thermal) line broadenings are observed in the cooler chromospheric / transition-region lines and they are localized near the site where the closed magnetic loops reconnect with the open magnetic field lines. Both enhanced broadenings are found in the sites with redshifts in the lines, surrounded by the region with blueshifts. One enhanced line broadening is associated with a small flare without energetic particle signatures while another occurs just after the particle acceleration signatures of the main flare terminated.

Conclusions. The observed excess broadening appears to be not directly related to the energetic ion production and motions. Further investigations where the critical impulsive phase of the flare is covered are required, ideally with high-resolution spectrometers intentionally pointed to the ${ }^{3} \mathrm{He}$-rich solar energetic particle source.
\end{abstract}

Key words. acceleration of particles - magnetic reconnection - techniques: imaging spectroscopy - Sun: particle emission - Sun: flares

\section{Introduction}

Impulsive (or ${ }^{3} \mathrm{He}$-rich) solar energetic particle (SEP) events are characterized by a peculiar ion composition, markedly different from the corona or solar wind (Mason 2007, and references therein). The heavy ion enhancement in the impulsive SEP events is an increasing function of ion mass. For example, Fe is enhanced by a factor of about 10 compared to its coronal abundance; while the enrichment of ultra-heavy (UH) ions readily exceed an enhancement factor of 100 . This pattern conflicts with the enhancement of low-mass ${ }^{3} \mathrm{He}$ that is enhanced by factors up to $10^{4}$. It is believed that the anomalous composition is the signature of a unique acceleration process operating at the flare sites. A resonant interaction with plasma waves has been commonly involved in models of ion acceleration and fractionation in solar flares (Fisk 1978; Winglee 1989; Temerin \& Roth 1992; Miller 1998; Zhang 2004; Liu et al. 2006; Kumar et al. 2017) though another, non-wave, mechanisms (scattering on magnetic islands or DC electric field) have been suggested (e.g., Drake et al.2009; Fleishman \& Toptygin 2013). Electrons are almost always accelerated to sub-relativistic speeds in these events as demonstrated by direct in-situ measurements (Reames et al. 1985; Wang et al. 2012) or by detection of type III radio bursts (Reames \& Stone 1986; Nitta et al. 2006).

${ }^{3} \mathrm{He}$-rich SEP events have been associated with minor (mostly B- and C-class) soft X-ray flares (Reames et al. 1988a;
Reames \& Ng 2004; Nitta et al. 2006, 2015; Bučík et al. 2014, 2016). In addition, an inverse correlation between the soft Xray peak intensity and ${ }^{3} \mathrm{He}(\mathrm{UH})$ enrichment has been reported (Reames et al. 1988a; Reames \& Ng 2004). ${ }^{3} \mathrm{He}$-rich flares have been commonly observed as a jet-like form in extreme ultraviolet (EUV; Nitta et al. 2006, 2015; Chen et al. 2015; Bučík et al. 2018) or white-light coronograph images (Kahler et al. 2001; Wang et al. 2006). The association with jets has been interpreted as an evidence for magnetic reconnection involving field lines open to interplanetary space (Shibata et al. 1992). Remote observations of ion acceleration in solar flares are quite limited. The energetic ions do not show clear flare signatures as energetic electrons do through the hard X-ray (HXR) bremsstrahlung or radio emissions. Gamma-ray nuclear lines, produced in a bombardment of energetic $\left(>1-10 \mathrm{MeV}\right.$ nucleon $\left.^{-1}\right)$ ions with ambient nuclei in the chromosphere, were observed only in major (Xor M-class) flares (e.g., Vilmer et al. 2011). Although the abundances derived from $\gamma$-ray lines show ${ }^{3} \mathrm{He}$ and heavy-ion enrichment (Murphy et al. 1991; Mandzhavidze et al. 1999), the $\gamma$-ray lines were generally not observed in ${ }^{3} \mathrm{He}$-rich SEP events (but see Van Hollebeke et al. 1990; Kartavykh et al. 2007, for the exceptions).

There are theoretical predictions for the formation of asymmetrical broadened profiles (extended red wings) in $\mathrm{H}_{\text {I }}$ Lyman$\alpha(1216 \AA)$ and He II Lyman- $\alpha$ (304 $\AA$ ) atomic lines (e.g., 
Orrall \& Zirker 1976; Canfield \& Chang 1985; Peter et al. 1990; Brosius \& Woodgate 1999) caused by the accelerated ions in solar flares. Specifically, the low-energy $\left(<1 \mathrm{MeV}\right.$ nucleon $\left.^{-1}\right)$ protons or $\alpha$-particles (invisible in $\gamma$-ray lines) can capture electrons due to charge-exchange with thermal neutral hydrogen in the chromosphere and radiate non-thermal Doppler-broadened Lyman- $\alpha$. The profiles are expected to extend rather far into red wings, $\sim 10 \AA$ for $\mathrm{H}$ I and $4-5 \AA$ for He II. Similar processes of charge-exchange between suprathermal $(\mathrm{keV})$ protons and residual neutral hydrogen in the corona have been discussed by Laming et al. (2013). The authors have suggested that the suprathermal ions should manifest themselves through extended wings in resonantly scattered chromospheric $\mathrm{H}_{\mathrm{I}}$ Lyman- $\alpha$ in the corona. However, so far, no clear evidence of Doppler-broadened Lyman- $\alpha$ from energetic ions in solar flares has been observed (Brosius 2001; Hudson et al. 2012) though the effect has been reported in a flare on the red dwarf star AU Mic (Woodgate et al. 1992). Brosius (2001) has examined C-class flare with a SOHO CDS spectrometer for a signal of enhanced red wings in the profile of He II Ly $\alpha$ line. Hudson et al. (2012) have examined nine hard X-ray $/ \gamma$-ray events (in $\mathrm{M}$ - or X-class flares) for signatures of broad wings in He II Ly $\alpha$ using the full-Sun EVE instrument aboard SDO. The authors discuss that strong chromospheric heating during the flare may reduce or mask the chargeexchange mechanism. Jeffrey et al. (2016, 2017) have analyzed the Fe XVI 262.98 $\AA$ and Fe xxIII 263.76 $\AA$ line profiles observed by the Hinode EIS in two X-class flares and found the profiles consistent with a kappa distribution of emitting ions with kappa values corresponding to the highly accelerated ion distribution. The authors have speculated that a flare accelerated Fe ions could account for the observed excess broadening.

A suitable candidate for EUV spectral line investigations, with a low background, may be ${ }^{3} \mathrm{He}$-rich SEP events whose solar sources have been associated with minor X-ray flares or weak EUV brightenings. In addition, in such events, we have direct evidence for ion acceleration in the flare via in-situ measurements of energetic ion fluxes. In this study, we present the first-time observations of EUV spectral lines in the source flare of ${ }^{3} \mathrm{He}$-rich SEPs.

\section{Methods}

We study the solar source of ${ }^{3} \mathrm{He}$-rich SEPs with the Coronal Diagnostic Spectrometer (CDS; Harrison et al. 1995) aboard Solar and Heliospheric Observatory (SOHO). The CDS consists of normal incidence (NIS) and grazing incidence (GIS) spectrometers. The observations examined in this study were obtained by NIS using a $4^{\prime \prime} \times 240^{\prime \prime}$ slit to produce a raster image. The NIS covers 307-379 $\AA$ and 513-633 $\AA$ wavelength ranges, with approximate spectral resolutions $0.32 \AA$ and $0.54 \AA$ full width at half-maximum (FWHM), respectively. The following three lines are examined: He I $584.30 \AA$, O v $629.39 \AA$, and Fe XVI $360.80 \AA$, corresponding to the formation temperatures $\sim 0.03 \mathrm{MK}, 0.25 \mathrm{MK}$, and $2.5 \mathrm{MK}$, respectively. These CDS lines have been analyzed in several previous studies on solar flares (e.g., Czaykowska et al. 1999; Milligan et al. 2006a.b; Teriaca et al. 2006). We remind that the He I stands for neutral helium, $\mathrm{O} v$ is $4 \times$ ionized oxygen with charge-to-mass ratio $Q / A=4 / 16$ and Fe XVI is $15 \times$ ionized iron with $Q / A=15 / 56$. We also examine EUV images of the ${ }^{3} \mathrm{He}$-rich solar source obtained by SOHO EIT (Delaboudinière et al. 1995) and the Transition Region and Coronal Explorer (TRACE; Handy et al. 1999), and magnetograms obtained by SOHO MDI (Scherrer et al.

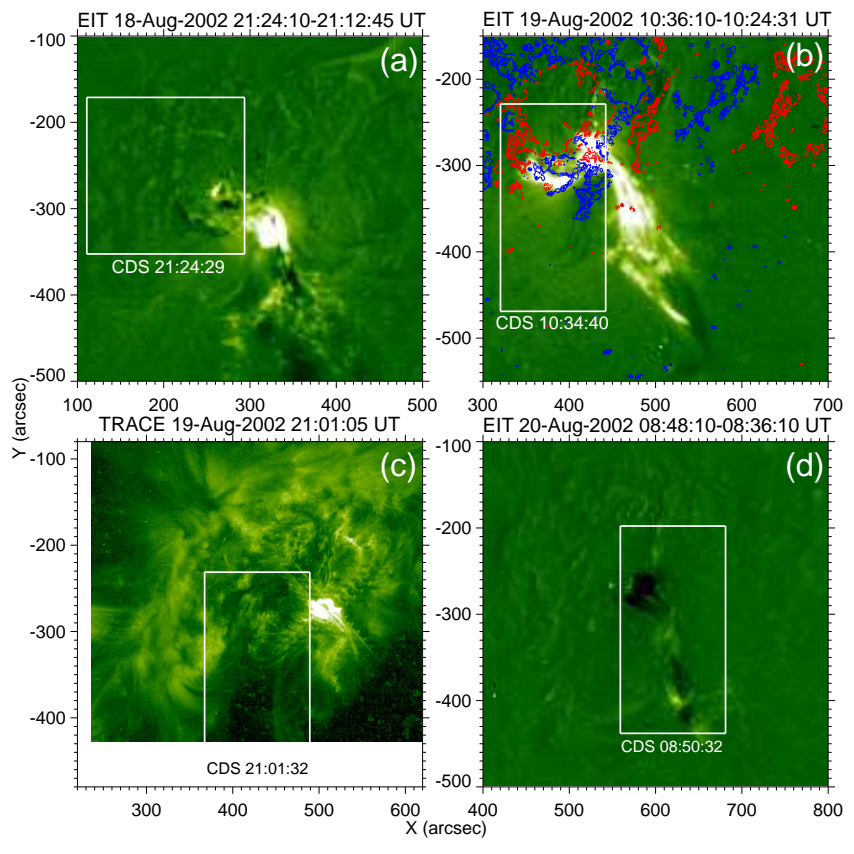

Fig. 1. SOHO EIT $195 \AA ̊$ EUV 12-min running differences images for flares in event 1 (panel a), event 2 (panel b) and event 4 (panel d). Overplotted in panel (b) are $\pm 500 \mathrm{G}$ SOHO MDI magnetogram data contours for 2002 August 19 11:11 UT, where blue/red is positive/negative polarity. TRACE $195 \AA$ EUV direct image (re-scaled to L1 and aligned with CDS Fe xvi $360.80 \AA$ line raster image) for event 3 where SOHO EIT data are not available (panel c). Note that empty area at bottom of the panel (c) is due to a restricted TRACE FOV. White rectangles depict the CDS FOV. While the images in events $1,2,3$ are near ( $\pm 1-2$ minutes) the flare maximum the image in event 4 is $\sim 20$ minutes after the flare maximum.

1995). The ${ }^{3} \mathrm{He}$-rich SEPs are analyzed using the measurements from SOHO Electron Proton Helium Instrument (EPHIN; Müller-Mellin et al. 1995). The EPHIN, a $d E / d x$ versus $E$ telescope, measures hydrogen and helium isotopes in the range $4 \mathrm{MeV}_{\text {nucleon }}{ }^{-1}$ to $>53 \mathrm{MeV}$ nucleon $^{-1}$. We also inspect dynamic radio spectra for the event associated type III radio bursts. The radio data are provided by the Wind WAVES instrument (Bougeret et al. 1995) with a frequency range $(<16 \mathrm{MHz})$ covering emission generated from about $2 R_{\odot}$ to 1 au and Phoenix2 (Bleien, Switzerland) broadband spectrometer (Messmer et al. 1999) operated in the frequency range $0.1-4 \mathrm{GHz}$. Furthermore, we make use of soft and HXR observations from NOAA GOES-10 XRS and Wind KONUS (Aptekar et al. 1995) sensors, respectively. The interplanetary magnetic field (IMF) and solar wind speed measurements were provided by the MAG (Smith et al.1998) and SWEPAM (McComas et al.1998) instruments on ACE. Note that while SOHO, ACE and Wind were in the interplanetary space the TRACE was in geocentric orbit.

To find a ${ }^{3} \mathrm{He}$-rich solar source in the CDS field-of-view (FOV), we inspect all (93) ${ }^{3} \mathrm{He}$-rich SEP events of previous solar cycle 23 with a reported solar source (Mason et al. 2002; Nitta et al. 2006; Wang et al. 2006). The solar sources in 67 events have been identified by Nitta et al. (2006), in another 19 events by Wang et al. (2006) and in 7 events by Mason et al. (2002). These events are from the period September 1997 to March 2003. Note that the CDS started scientific operation in April 1996. To identify the solar source of a ${ }^{3} \mathrm{He}$-rich SEP event, Wang et al. (2006) examined $\mathrm{H} \alpha$ and SOHO EIT flares within a few hours of the estimated ion injection time; Nitta et al. (2006) examined type III radio bursts in the 5-hour window preced- 
ing the observed ion onset and then searched for the associated brightening in SOHO EIT coronal images; and Mason et al. (2002) selected the event associated X-ray flare based on the estimated ion injection time. Note that overlays of CDS FOV with other imaging instruments on $\mathrm{SOHO}$ are subject to a $5^{\prime \prime}$ pointing uncertainty of the CDS (Fludra 2001).

We found only four ${ }^{3} \mathrm{He}$-rich SEP source flares fully or partially covered by the CDS FOV. All these flares were from the same active region, marked by NOAA number 10069. Fig. 1 shows SOHO EIT or TRACE EUV images of these ${ }^{3} \mathrm{He}$-rich SEP sources with superimposed CDS FOVs. Fig. 11 and Fig. 1, indicate that the flares in event 1 and 3 were only partially captured with CDS. The flare in event 2 was quite well captured (see Fig. 1b) and therefore is further investigated in detail. In event 4 only the post-flare phase was captured (see Fig. 1 d). Note that a dimming jet exhibited an interesting twisted configuration in event 4 . Table 1 shows some characteristics of these flares and the associated particle events. Column 1 gives the event number, column 2 the particle event start time, and column 3 the type III radio burst start time. Columns 4, 5, 6, and 7 indicate the GOES X-ray flare start time, time at the flare maximum, the flare location, and class, respectively. Columns 8 and 9 provide the ${ }^{3} \mathrm{He} /{ }^{4} \mathrm{He}$ and $\mathrm{Fe} / \mathrm{O}$ abundance ratios, respectively. Table 2 lists some parameters of CDS studies that match these ${ }^{3} \mathrm{He}$-rich flares. Column 1 gives the event number, column 2 the start time of the raster image, column 3 the duration of the rastering, column 4 the temporal resolution, and column 5 the exposure time. Columns 6,7 , and 8 indicate the CDS pointing, FOV, and the spatial resolution, respectively. Columns 9 and 10 indicate the names of the CDS studies and their total durations, respectively. Note that the observational mode in event 2 includes four lines $\mathrm{He}$ I, O v, Mg Ix, and Fe Xvi.

\section{Results}

Events 1-4 have been included in previous studies (see references in Table 1). All these events were associated with M-class flares. The events exhibited large ion intensities at high energies $\left(>10 \mathrm{MeV}\right.$ nucleon $\left.{ }^{-1}\right)$ and therefore called large impulsive SEP events (Reames \& Ng 2004) as opposed to more typical small impulsive events. The events are electron-rich (or proton-poor) as reported in a study of Cane et al. (2010). The events showed large enhancements of trans-Fe elements by a factor of $10^{2}-10^{3}$ for $Z>50$ (Reames \& Ng 2004). The measured abundances in events $2-4$ are consistent with a coronal temperature $2.5-3 \mathrm{MK}$ in their source (Reames et al. 2014). Wiedenbeck et al. (2010) have performed a detailed elemental and isotopic compositional analysis of the measurements above $10 \mathrm{MeV}$ nucleon $^{-1}$ in event 4 (2002 August 20). Based on the inferred charge states (assuming $Q / A$-dependent fractionation) a best-fit temperature in source plasma was found to be $\sim 4 \mathrm{MK}$ with large deviations to higher temperatures for $\mathrm{Fe}-\mathrm{Ni}$. The authors also reported a temperature $\sim 1.6 \mathrm{MK}$, assuming a possible $Q^{2} / A$ law. They also reported observed Fe charge states $Q$ between 18 (Fe xIx) and 22 (Fe xxIII) at low energies $\left(0.25-1 \mathrm{MeV}\right.$ nucleon $\left.{ }^{-1}\right)$. The $2002 \mathrm{Au}-$ gust 20 event was the largest impulsive event in solar cycle 23 observed at energies $>10 \mathrm{MeV}$ nucleon $^{-1}$ (Leske et al. 2003).

The EUV flare type in the investigated event 2 has been noted as an ejection in $195 \AA$ SOHO EIT images in Wang et al. (2006). Examining the TRACE $195 \AA$ images, the flare type in all four events 1-4 has been noted as a jet in Nitta et al. (2006). The SOHO EIT flare in event 2 occurred close to a new emergence of a negative-polarity flux observed with SOHO

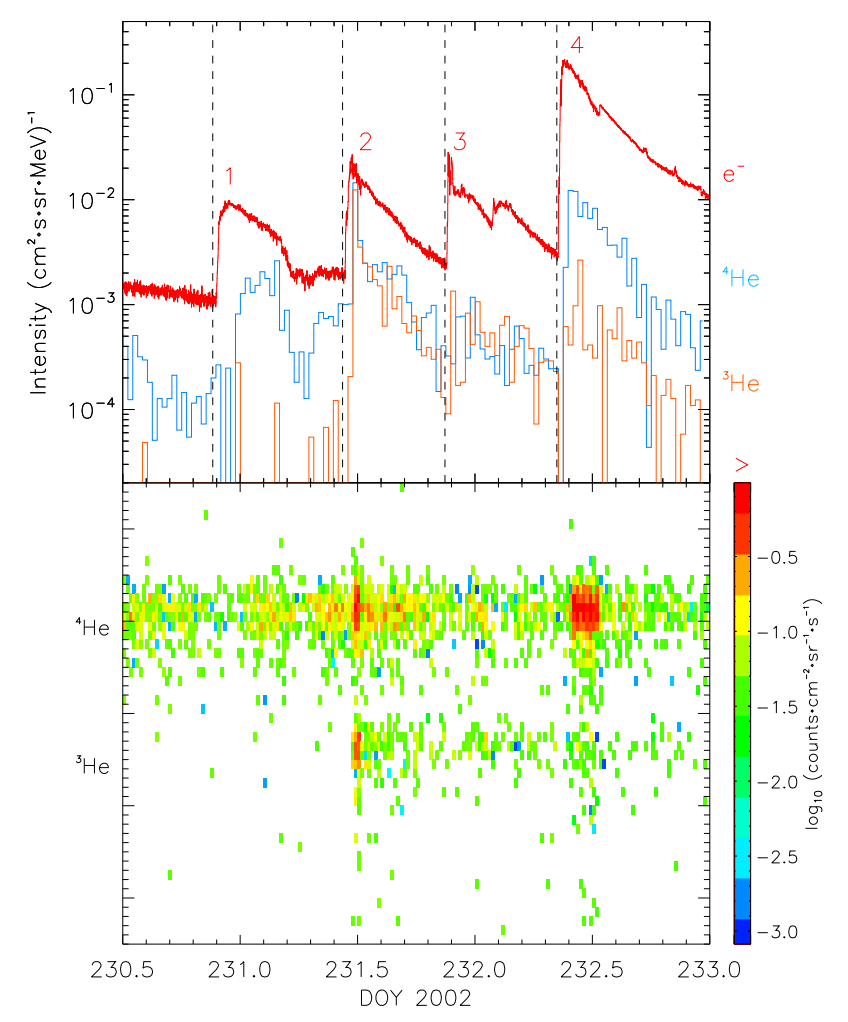

Fig. 2. SOHO EPHIN measurements. Top: 1-min averaged 0.7$3.0 \mathrm{MeV}$ electron intensity (divided by 500) and 30-min averaged 10$25 \mathrm{MeV}$ nucleon ${ }^{-1}{ }^{3} \mathrm{He},{ }^{4} \mathrm{He}$ intensities. Dashed vertical lines indicate X-ray flares associated with events 1-4 (see Table 1). Bottom: helium mass spectrogram of individual PHA ions with no energy restriction.

MDI magnetograms between 2002 August 19 01:36-11:25 UT (Tan \& Yan 2008). Using the Nançay Radio-Heliograph observations at $164 \mathrm{MHz}$, Tan \& Yan (2008) found that the metric type III radio bursts were located near the top of the loops at height $\sim 0.15 R_{\odot}$. Events 3 and 4 have been included in a study on electron acceleration in solar jets (Krucker et al. 2011). A HXR imaging with RHESSI showed three chromospheric (HXR) sources in event 4, two at the loop foot-points and one related to the open field line. In event 3 only two chromospheric sources were seen, where a third source could be hidden by the extended flare (Krucker et al. 2011). The authors found the onset of the EUV jets observed with TRACE coincident with the HXR emission. For event 2 no RHESSI solar data are available for the associated flare at 10:07-10:41 UT on 2002 August 19.

The PFSS (potential-field source-surface) extrapolations of the photospheric magnetic field (National Solar Observatory synoptic maps) in event 2 show the Earth-directed open field lines near the associated flare (Wang et al. 2006). Specifically, the field lines that cross the source surface at longitudes between W35 and W65 and at latitudes less than $20^{\circ}$ from the ecliptic (but not those directed to the ecliptic) were rooted next $\left(<4^{\circ}\right)$ to the event source. Wang et al. (2006) have noticed that a dipole field line that intersects the source surface at $20^{\circ}$ would continue to bend equatorward beyond the source surface. The PFSS extrapolations based on the MDI magnetograms assimilated into the evolving surface flux model show no open field lines within $10^{\circ}$ from the flare location (Nitta et al. 2006). Using the Wilcox Solar Observatory synoptic maps the authors report open field lines with a combined offset at the photosphere (from the associated flare) and at the source surface (from the Parker spiral footpoint) 
Table 1. ${ }^{3} \mathrm{He}$-rich SEP event properties

\begin{tabular}{|c|c|c|c|c|c|c|c|c|c|}
\hline \multirow[t]{2}{*}{ Event } & \multirow[t]{2}{*}{ SEP start ${ }^{a}$} & \multirow{2}{*}{$\begin{array}{c}\text { Type III } \\
\text { start }^{b}\end{array}$} & \multicolumn{4}{|c|}{ 1-8 Å GOES X-ray flare ${ }^{c}$} & \multirow[t]{2}{*}{${ }^{3} \mathrm{He} /{ }^{4} \mathrm{He}^{d}$} & \multirow[t]{2}{*}{$\mathrm{Fe} / \mathrm{O}^{e}$} & \multirow[t]{2}{*}{ Ref. } \\
\hline & & & Start & Max & Location & Class & & & \\
\hline 1 & 2002-Aug-18 23:00 & & $21: 12$ & $21: 25$ & S13V & $\mathrm{M} 2.2$ & $0.02 \pm 0.01$ & $0.40 \pm 0.04$ & $1,2,3$ \\
\hline 2 & & & & & & & & & $1,2,3,4,5,6,7$ \\
\hline 3 & 2002-Au & & $20: 56$ & 21:02 & S11 & M3.1 & 0.08 & 0.07 & $1,2,7,8$ \\
\hline 4 & 2002-Aug-20 10:00 & $08: 25$ & $08: 22$ & $08: 26$ & S11W38 & M3.4 & $0.06 \pm 0.02$ & $1.96 \pm 0.04$ & $1,2,3,4,7,8,9$ \\
\hline
\end{tabular}

Notes. ${ }^{(a)}$ Wind LEMT 2-3 MeV nucleon ${ }^{-1}$ (Nitta et al. 2006) ${ }^{(b)}$ Wind WAVES 4 kHz-13.8 MHz (Nitta et al. 2006) ${ }^{(c)}$ from Nitta et al. (2006) but start time (Start) and time at flux maximum (Max) from Solar and Geophysical Event Reports compiled by the NOAA Space Weather Prediction Center (SWPC); ftp://ftp. Swpc .noaa.gov/pub/warehouse/2002/(d) SOHO EPHIN 5-25 MeV nucleon ${ }^{-1}$ (this work) ${ }^{(e)}$ Wind LEMT 3.3$10 \mathrm{MeV}$ nucleon $^{-1}$ (Reames \& Ng 2004)

References. (1) Nitta et al. (2006); (2) Reames \& Ng (2004); (3) Cane et al. (2010); (4) Leske et al. (2003); (5) Wang et al. (2006); (6) Tan \& Yan (2008); (7) Reames et al. (2014); (8) Krucker et al. (2011); (9) Wiedenbeck et al. (2010)

Table 2. SOHO CDS data for ${ }^{3} \mathrm{He}$-rich SEP sources

\begin{tabular}{|c|c|c|c|c|c|c|c|c|c|}
\hline \multirow{2}{*}{$\begin{array}{l}\text { SEP } \\
\text { event }\end{array}$} & \multirow[t]{2}{*}{ Raster start time } & \multirow{2}{*}{$\begin{array}{l}\text { Dur. } \\
(\mathrm{sec})\end{array}$} & \multirow{2}{*}{$\begin{array}{c}\text { Tem. res. } \\
(\mathrm{sec})\end{array}$} & \multirow{2}{*}{$\begin{array}{l}\text { Exp. time } \\
\text { (sec) }\end{array}$} & \multirow{2}{*}{$\begin{array}{l}\text { Pointing } \\
\text { (arcsec) }\end{array}$} & \multirow{2}{*}{$\begin{array}{c}\text { FOV } \\
(\operatorname{arcsec})\end{array}$} & \multirow{2}{*}{$\begin{array}{c}\text { Spatial res. } \\
(\operatorname{arcsec})\end{array}$} & \multicolumn{2}{|c|}{ CDS study $^{a}$} \\
\hline & & & & & & & & Name & Dur. \\
\hline 1 & 2002-Aug-18 21:24:29 & 681 & 15.1 & 8 & $202.26,-261.81$ & $182.80 \times 181.40$ & $4.06 \times 3.36$ & FLARE_AR & 8.4 \\
\hline 2 & 2002-Aug-19 10:34:40 & 318 & 10.6 & 5 & $381.22,-348.87$ & $121.90 \times 240.20$ & $4.06 \times 1.68$ & CD5 & 4.8 \\
\hline 3 & 2002-Aug-19 21:01:32 & 321 & 10.7 & 5 & $428.32,-351.31$ & $121.90 \times 240.20$ & $4.06 \times 1.68$ & CD5 & 10.1 \\
\hline 4 & 2002-Aug-20 08:50:32 & 318 & 10.6 & 5 & $620.10,-317.88$ & $121.90 \times 240.20$ & $4.06 \times 1.68$ & CD5 & 2.2 \\
\hline
\end{tabular}

Notes. ${ }^{(a)}$ FLARE_AR: Flaring Active Region Study, CD5: Coronal Dynamics; Dur. (hr)

of less than $10^{\circ}$. It has been pointed out that the PFSS extrapolated field depends to some extent on the input magnetograms (Nitta et al. 2006).

\section{1. ${ }^{3} \mathrm{He}$-rich SEP events $1-4$}

Due to a limited element resolution of the Wind LEMT instrument, only an upper limit of 0.2 has been obtained for the ${ }^{3} \mathrm{He} /{ }^{4} \mathrm{He}$ ratio in events $1-4$ at $2.1-2.5 \mathrm{MeV}$ nucleon $^{-1}$ (Reames \& Ng 2004). Fig. 2] shows SOHO EPHIN measurements of ${ }^{3} \mathrm{He}$-rich SEP events $1-4$ in the period 2002 August 18 (day 230) 12:00 UT - August 21 (day 233) 00:00 UT. The ions with a parallel incidence are selected that improves the isotopic resolution, though at the cost of decreased statistics. Note on the ${ }^{3} \mathrm{He}$ increase near the detection limit in event 1 . The upper panel of Fig. 2 shows the $0.7-3.0 \mathrm{MeV}$ electron intensity, and the $10-25 \mathrm{MeV}$ nucleon ${ }^{-1}{ }^{3} \mathrm{He}$ and ${ }^{4} \mathrm{He}$ intensities; the lower panel shows the helium mass spectrogram. All four ${ }^{3} \mathrm{He}$-rich SEP events were accompanied by relativistic electron events. The examined event 2 shows the highest ${ }^{3} \mathrm{He}$ intensity and a large ${ }^{3} \mathrm{He}$ enrichment. A possible ion event associated with the second electron increase in event 3 is not included in ${ }^{3} \mathrm{He} /{ }^{4} \mathrm{He}$ in Table 1.

The electron onset time in event 2 at the energy range $0.25-$ $0.7 \mathrm{MeV}$ is measured at $10: 45 \mathrm{UT} \pm 1 \mathrm{~min}$. Assuming a scatterfree transport along the nominal Parker spiral (length 1.1 au for a measured solar wind speed $\sim 500 \mathrm{~km} \mathrm{~s}^{-1}$ ), the propagation time for electrons with a mean energy $0.42 \mathrm{MeV}$ (speed $0.84 c$ ) would be 11 minutes. It implies the solar release time around 10:34 UT. Subtracting a photon travel time of $8.3 \mathrm{~min}$, the type III radio bursts start time at the Sun is at 10:22 UT in event 2 (see Table 1). Thus, the inferred release of relativistic electrons is delayed by $12 \mathrm{~min}$ from the type III bursts. A longer travel distance and/or scattering during the transport may be responsible for a delay (e.g., Kahler et al.2007). Because of several assumptions, the type III radio burst observations provide a more reli- able measurement of the electron release time than the estimate based on electron propagation time. There is no significant proton increase associated with event 2 , instead, the event is clear at the helium channels. To obtain an accurate timing for ions is difficult because of low statistics, further complicated by the high particle flux which increases background and triggers the instrument geometric factor into a smaller one at 11:14 UT on August 19. Using the pulse-height analyzed (PHA) data and selecting ${ }^{3} \mathrm{He}$ in a narrow band between 10 and $15 \mathrm{MeV}$ nucleon $^{-1}$, the estimated onset time is 11:35 $\pm 10 \mathrm{~min}$. Assuming a mean energy around $12.2 \mathrm{MeV}$ nucleon $^{-1}$ implies a solar release time at $10: 38 \pm 10 \mathrm{~min}$, which would be compatible with the electron release time at 10:34 UT.

\subsection{Imaging observations of the source in event 2}

Fig. 3 shows the $122^{\prime \prime} \times 240^{\prime \prime}$ CDS raster images in O v $629.39 \AA$ line taken during different phases of the ${ }^{3} \mathrm{He}$-rich flare in event 2 . The rasters were built up by scanning the $4^{\prime \prime} \times 240^{\prime \prime}$ slit from the solar west to the east over 30 pointing positions in 5 minutes and $\sim 19$ seconds. The rasters in the upper panels display the average intensity in the line profile, in middle-row panels the line shift relative to the median wavelength obtained from the whole raster and in the bottom panels the line FWHM. After the SOHO temporary loss in 1998, the CDS spectral line profiles were found to have changed. To obtain the line shift and width from postrecovery data, we perform a single broadened Gaussian leastsquares fit to the line intensity profile in every pixel of the raster. W. T. Thompson (1999) reports a broadened Gaussian function that matches the post-recovery line profiles well. The broadened Gaussian function has two extra parameters to fit the asymmetry wings of the CDS instrumental response. The CDS intensity maps in the upper row of Fig. 3 indicate two distinct bright-

\footnotetext{
1 See CDS Software Note No. 53 (http://solar.bnsc.rl.ac.uk/ swnotes/cds_swnote_53.pdf)
} 


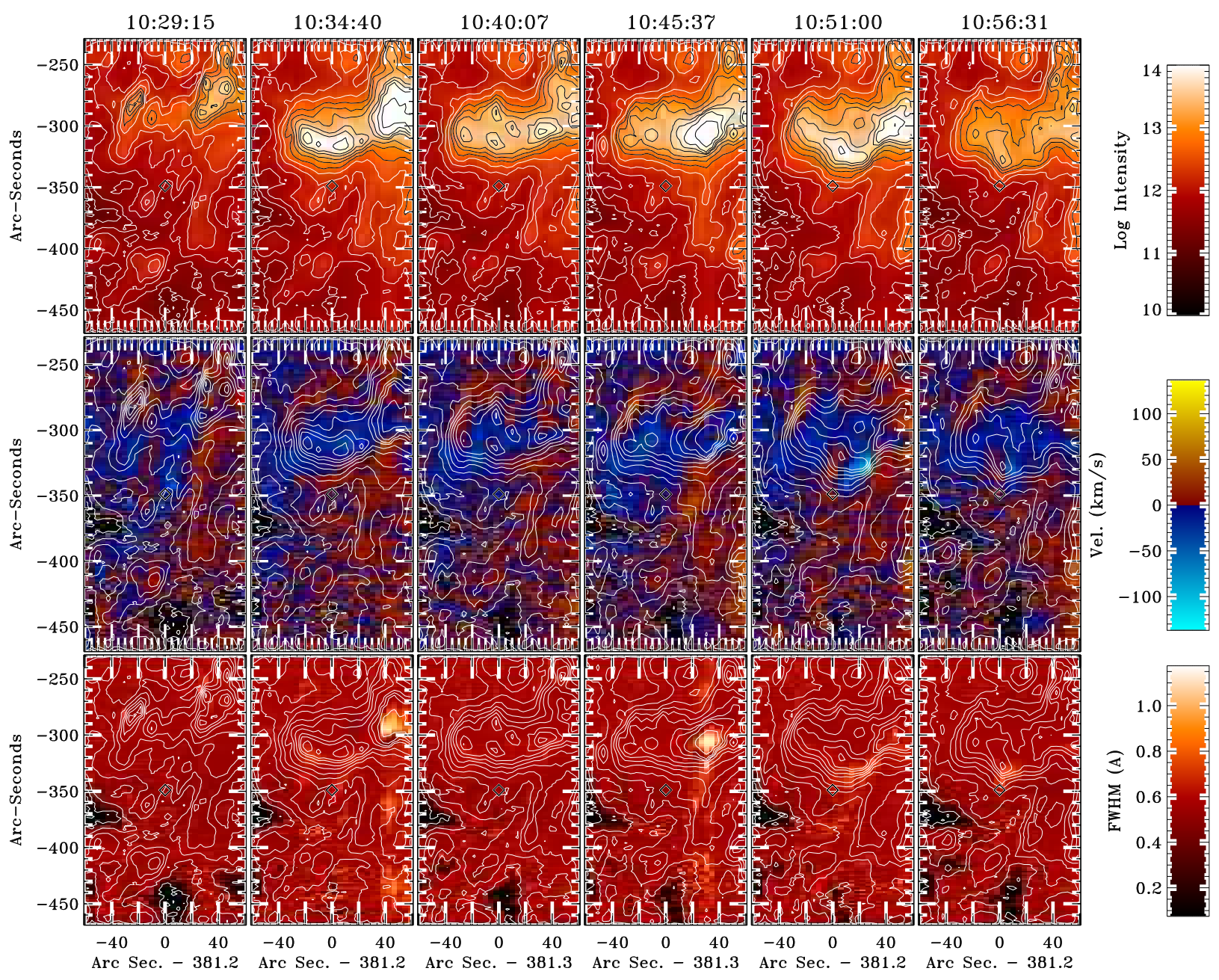

Fig. 3. The CDS rasters (O v $629.39 \AA$ Alne) of the source flare in event 2 between 10:29:15 and 10:56:31 UT on 2002 August 19. Times are given for the beginning of the rastering. Top row: intensity (photons $\mathrm{s}^{-1} \mathrm{~cm}^{-2} \mathrm{sr}^{-1}$ ). Middle row: wavelength shift relative to the median wavelength taken from the full image. Bottom row: the FWHM of the line. Over-plotted are the contours for every $0.25 \operatorname{lof}_{10} \log _{10}$ intensity.

enings, one at 10:34:40 UT and another at 10:45:37 UT. These intensity brightenings contain small areas of the enhanced line broadenings as seen in the bottom row of Fig. 3. The middle-row panels of Fig. 3indicate that both intensity brightenings roughly correspond to the blueshift (or plasma up-flow) region, though the enhanced line broadenings contain the redshift (down flow) areas or areas with the reduced up-flow velocity (dark blue pixels). The strongest blue shift is seen at 10:51:00 UT, just after the second brightening, and it is not related to the event associated flare (see Fig. 77).

Fig. 4 depicts the broadened Gaussian fits to the Ov line intensity profiles for one of those pixels showing the strong line broadening at 10:34:40 UT and 10:45:37 UT. The quiet line profiles outside the flaring region are also shown. These have widths comparable to the instrumental width. Fig. 5 shows the CDS raster images for He I 584.30 $\mathrm{A}$ and Fe XVI $360.80 \AA$ lines at times when the strong $\mathrm{O} v$ line broadenings are seen. Similarly to $\mathrm{O} v$ the $\mathrm{He}_{\mathrm{r}}$ line is fitted with a single broadened Gaussian function. Since there is a second weaker line on the blue side of the Fe XVI peak (a blend of Fe XIII lines at $359.6 \AA$ and $359.8 \AA$ ) the Fe XVI line is fitted with a double Gaussian where the FWHM is taken from the stronger central line. The corresponding line broadenings are seen in cooler He I line, but not in the hot Fe XVI line. The He I line broadenings at 10:34:40 and 10:45:37 UT are observed in the regions with the plasma down-flows. It is consis-
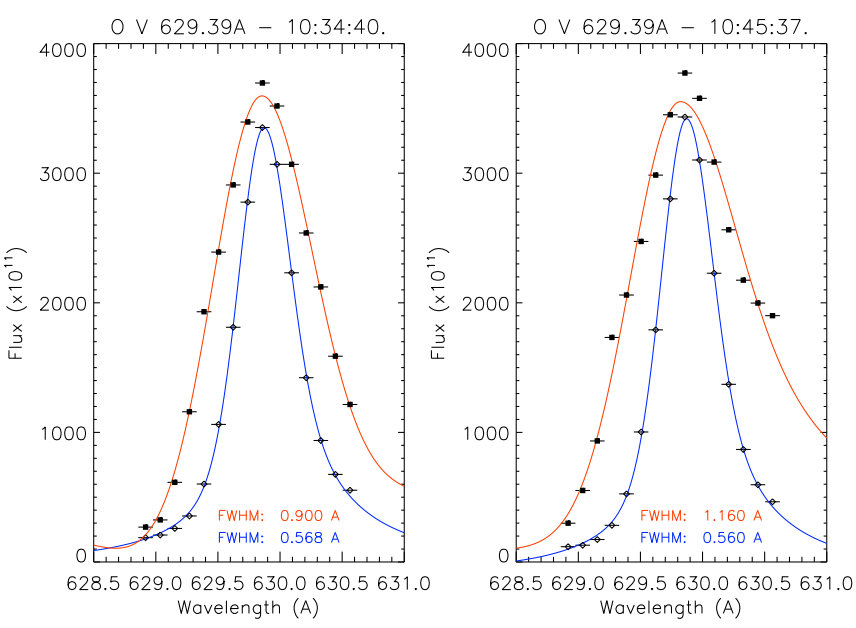

Fig. 4. Left: Broadened Gaussian fits to the $\mathrm{O} v$ intensity (photons $\mathrm{s}^{-1} \mathrm{~cm}^{-2} \mathrm{sr}^{-1}$ ) from the pixel $\left(420^{\prime \prime},-290^{\prime \prime}\right)$ (red curve) and to the intensity summed over 90 pixels (to get a similar level of counts) around $\left(370^{\prime \prime},-420^{\prime \prime}\right)$ (blue curve) at 10:34:40 UT on August 19. Right: Similar to the left panel, but at 10:45:37 UT on August 19, and the red curve is for the pixel $\left(410^{\prime \prime},-310^{\prime \prime}\right)$.

tent with the observations in Ov line, though the plasma flows are more mixed in $\mathrm{O} v$ line. 

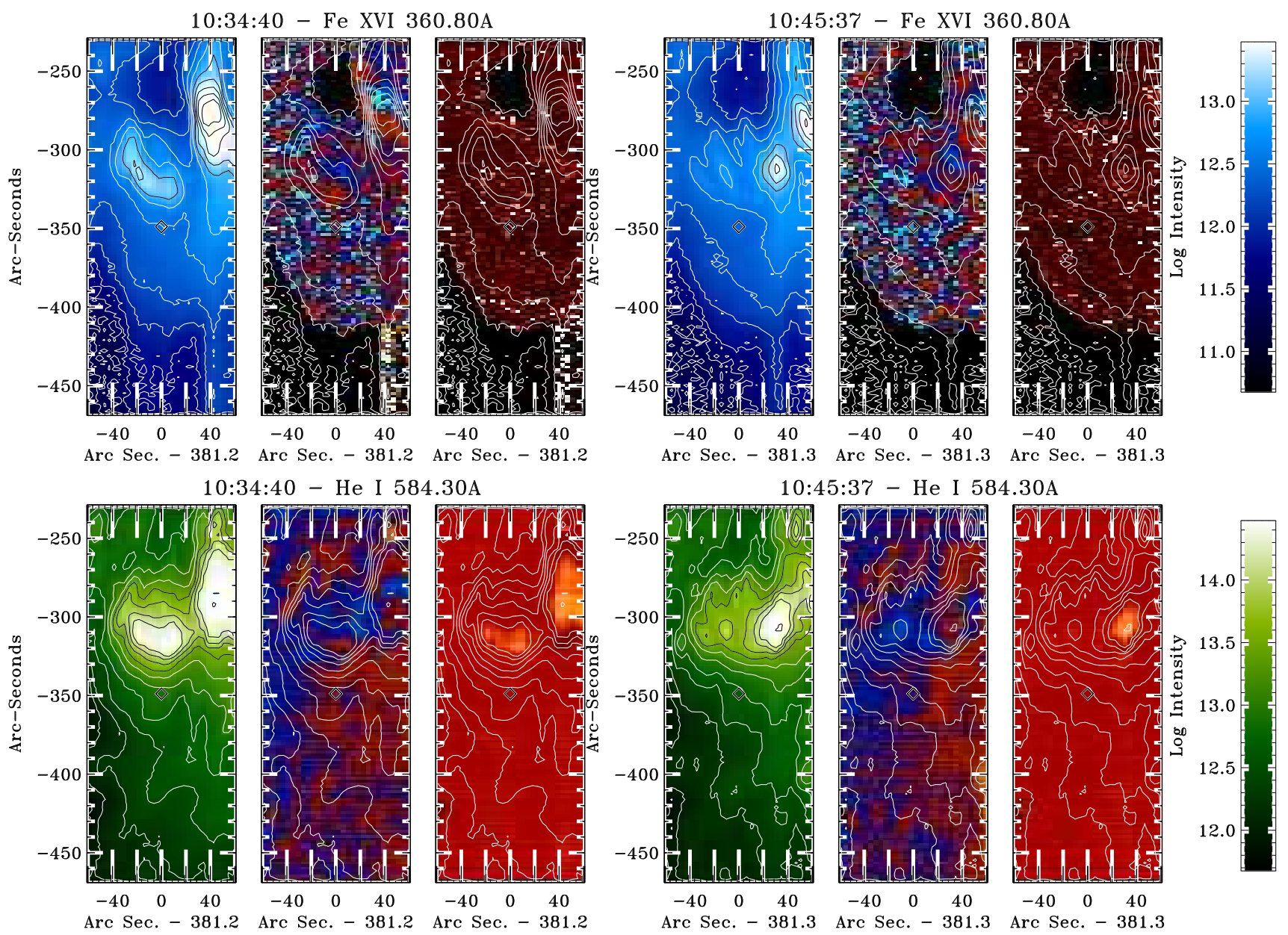

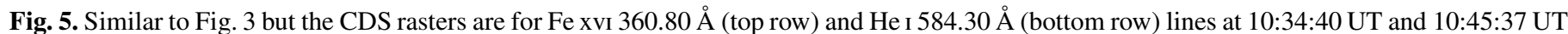
on 2002 August 19. Places in the Fe xvi image that were below a certain intensity threshold have not been fitted. The velocity and FWHM panels use the same colour tables as Fig. 3

The intensity brightening at 10:34:40 UT occupies two separate areas. As indicated in the EIT image ( $5^{\prime \prime}$ spatial resolution) in Fig. 1b the brighter area on the west corresponds to the jet locus and the less bright region on the east to the top of the closed loop. The locus of the jet originated at the southwest boundary of a large sunspot at the interface with the minor polarity field. Note that the negative IMF polarity during the event 2 is consistent with sunspot polarity field. To see more details of the jet we plot in Fig. 6 the high-resolution (1") TRACE 195 EUV images at times of the enhanced line broadenings. The pixels with the line broadening at 10:34:40 UT coincide with the jet locus and the area where the coronal closed structure reconnects with the open field lines. During the second line broadening at 10:45:37 UT a flare/small-jet was observed shortly between 10:45-10:50UT with the TRACE high cadence $(17 \mathrm{sec})$ EUV images. The post flare arcade of the closed loops is well seen in the upper-right part of the image in Fig. 6b.

To align between TRACE and SOHO CDS we at first check the inclinations of different flaring structures in SOHO EIT $195 \AA$ Amage on 2002 August 19 10:36:10 UT (Fig. 1 1 b) and TRACE $195 \AA$ Å image on 2002 August 19 10:34:54 UT (Fig. 6r). Note that the time offset of 75 seconds between these two images corresponds to a negligible $0.1^{\prime \prime}$ in the solar rotation. The alignment was done after rescaling TRACE to L1. It is obvious that no significant rotation is needed to co-align the jet seen in both images. In the further step the TRACE $195 \AA$ images in Fig. 6) were
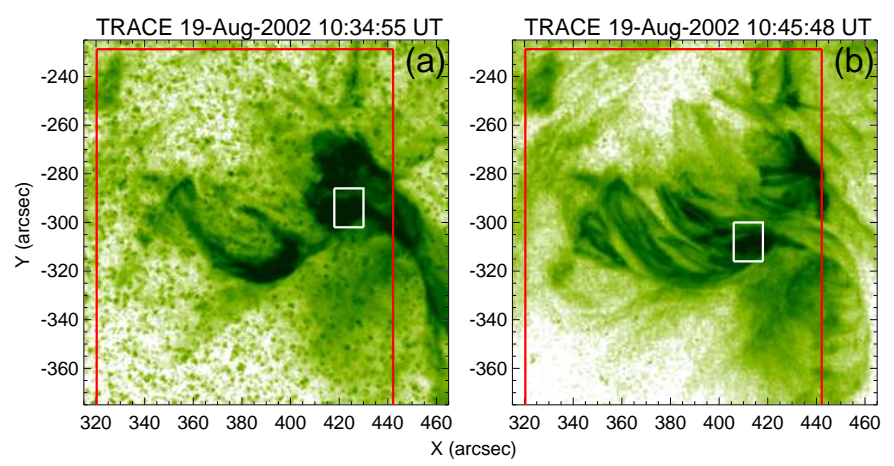

Fig. 6. TRACE $195 \AA$ EUV direct images (with the reverse color scale where dark corresponds to high intensities) of the source M2.1 flare in event 2 around the flare maximum (panel a) and ten minutes later (panel b). Red rectangles depict the CDS FOV. White rectangles mark the pixels with the enhanced line broadenings. Note that panel (a) shows in a better resolution the EIT flare in Fig. 1 $\mathrm{b}$. The noise spikes in the images are not filtered out.

manually shifted in solar $\mathrm{X}$ by $+10^{\prime \prime}$ to co-align with features observed in CDS Fe xvi 360.80 A line raster image (Fig. 5). The similar approach was applied to the TRACE image in Fig. 11. The EIT $195 \AA$ A channel includes emission from the bright Fe XII line formed at $\sim 1.6 \mathrm{MK}$ and therefore the comparison with CDS $2.5 \mathrm{MK} F \mathrm{xvI}$ line is reasonable. 


\section{3. $X$-ray and radio observations in event 2}

Fig. 7 7 a-b) shows GOES X-ray flux (1.0-8.0 ̊, 0.5-4.0 ̊) light curves and Wind WAVES radio spectrogram during the M2.1 class flare in event 2 . A strong type III radio burst was observed during the impulsive (or rapid X-ray flux increasing) phase of the flare. The line broadening observed at the raster 10:34:40 UT (marked by a leftmost solid vertical line) corresponds to the time just after the flare maximum and coincides with the termination of the type III radio burst (or a termination of energetic electron injection into interplanetary space). More accurately, the pixels with the enhanced line broadening were scanned even slightly later at $\sim 10: 35: 10-10: 35: 40$ UT. The same area was scanned in the previous rastering, around the flare (and type III burst) onset time at $\sim 10: 29: 45-10: 30: 15 \mathrm{UT}$, but not yet enhanced line broadening is seen. The excess line broadening at the raster 10:45:37 UT (marked by another vertical solid line) most likely corresponds to another X-ray flare, not well resolved from a high background during the decay phase of M2.1 flare. We can see that the GOES X-ray flux in the M2.1 flare does not continue decreasing to the background values, but forms a plateau around 10:45 UT that may correspond to the $<\mathrm{C} 2.5$ class flare. There were no type III radio bursts associated with this second line broadening. Either the jet might have formed along the closed magnetic field lines and/or the energy release was too weak for a particle acceleration.

After examining full-disk EIT difference images (12-min cadence) we note that the follow up C3.2 X-ray flare with a maximum at $\sim 10: 52$ UT on 2002 August 19 (see Fig. 7 h) originated in the same active region. Again, no type III radio bursts were accompanied by this flare. The low energy electrons $(<30 \mathrm{keV})$, attributed to the source of type III radio bursts Krucker et al. 1999), have been measured in event 2 by Wind 3DP telescope (Lin et al. 1995) in eight energy bins between 1.8 and $19 \mathrm{keV}$. However, due to a large pre-event background, the onset of the low-energy electron event is not well measured.

Fig. 7(c-d) shows high-resolution HXR and radio data in a 9-minute period, marked by a shaded bar in Fig. 7 (a-b), covering the impulsive and early decay phase of the M2.1 flare in event 2. The HXR intensity was detected by the Wind KONUS X-ray instrument in two energy channels $\sim 20-80$ and $\sim 80-300 \mathrm{keV}$. Multiple HXR bursts were detected between 10:30:40 and 10:34:00UT on August 19 (see also Wind KONUS Solar Flare database at http://www.ioffe. $\mathrm{ru} / \mathrm{LEA} / \mathrm{kwsun} /$ ). The radio spectra, in the frequency range 0.1-1.0 GHz, were observed with a Phoenix-2 Radio Telescope. A large group of intense type III bursts at 10:30:06-10:33:54 UT coincides with HXR bursts indicating that their parent energetic electrons are related to the same acceleration episodes. The NOAA NGDC Solar Radio Bursts Report (ftp:// ftp.ngdc .noaa.gov/STP/space-weather/solar-data/ solar-features/solar-radio/radio-bursts/reports/ spectral-listings/2002/) lists a large group of decimetric (DCIM) bursts $(>10)$ at 10:30:18-10:34:06UT in the frequency range $2.0-4.5 \mathrm{GHz}$ (Ondřejov). Just above the range of the WAVES instrument the type III bursts continue in the range $20-100 \mathrm{MHz}$ observed with Artemis-IV (Thermopylae) radiospectrograph.

\section{Discussion and further prospects}

The spectral line broadening is usually caused by thermal ion motion. In solar flares the excess (non-thermal) line broadening has been often observed (e.g., Fludra et al. 1989). It has been
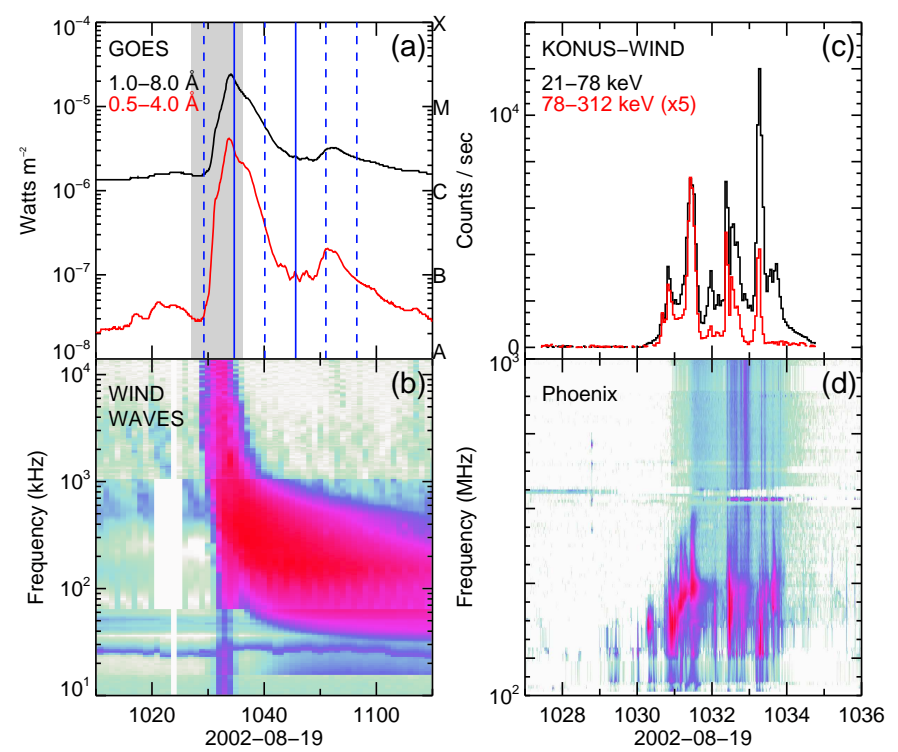

Fig. 7. (a) Full-disk GOES-10 3-sec X-ray fluxes. The labels A, B, C, $\mathrm{M}$, and $\mathrm{X}$ on right $\mathrm{y}$-axis indicate flare classes in 1-8 $\mathrm{A}$ channel. The vertical lines mark the start times of six CDS rasters; two solid vertical lines indicate the starts of the rasters when the excess line broadening is observed. (b) Wind WAVES 1-min averaged radio spectra. (c) Fulldisk Wind KONUS 3-sec averaged X-ray counts ${ }^{-1}$ in a 9-minute period marked by a shaded bar in panel (a). The $78-312 \mathrm{keV}$ fluxes are multiplied by a factor of 5. (d) Phoenix $100 \mathrm{~ms}$ radio spectra.

discussed that the excess broadening observed in EUV spectral lines may be an indicator of unresolved plasma flows (e.g., Milligan 2011), wave turbulence (e.g., Moortel \& Nakariakov 2012) or accelerated non-thermal ion motions (e.g., Jeffrey et al. 2016).

Jeffrey et al. (2017) have reported that a velocity distribution emitting from the FexvI $(Q / A=15 / 56)$ line has a higher excess broadening than the Fe XxIII $(Q / A=22 / 56)$ velocity distribution. This would be consistent with the measured heavyion enhancement that increases with low $Q / A$ ratio in ${ }^{3} \mathrm{He}$ rich flares (Reames \& Ng 2004; Mason et al. 2004) as well as with the mechanism of ion acceleration in reconnection exhausts (Drake et al. 2009) or cyclotron resonance with Alfvén waves (Kumar et al. 2017) that predict more efficient acceleration for ions with low $Q / A$. Note that $\mathrm{O} v$ and $\mathrm{Fe} x \mathrm{x}$ I have almost identical $Q / A$ and therefore should exhibit similar enhancement factor. However, no excess broadening was observed in Fe xvi line in the examined event. Jeffrey et al. (2017) have found that broadened line profiles may arise from non-thermal $\mathrm{Fe}$ if ions are locally accelerated on timescales $<0.1 \mathrm{~s}$. Due to these stringent conditions, the authors suggest that more plausible explanation for the observed line profiles are non-Gaussian turbulent velocities that was supported by the observations of small redshifts. Similarly, we observe small redshift at sites with the excess line broadening that could drive plasma turbulence responsible for the $\mathrm{He}_{\mathrm{I}}$ and $\mathrm{O} v$ line profiles.

Orrall \& Zirker (1976) have shown that non-thermal neutral hydrogen $\mathrm{H}$ I may become excited and radiate Ly $\alpha$. The authors pointed out that the effect can occur in the lines of other ions and atoms. Peter et al. (1990) have extended the calculations for a singly ionized helium (He II). The authors give the chargeexchange cross sections for formations of He II from $\alpha$-particle and of neutral $\mathrm{He}$ I from He II. One can find that a probability of double charge-exchange process to form $\mathrm{He}$ I rapidly decreases with the energy increase in 50-400 keV nucleon ${ }^{-1}$, implying that 
the effect would not be strong in the He I line; but details on a formation of broadened line profiles from the energetic He r are unknown.

It has been thought that ions in ${ }^{3} \mathrm{He}$-rich SEP events are accelerated at the impulsive phase of solar flares along with nonrelativistic electrons (Reames et al. 1985; Wang et al. 2012); Reames (1988b) has suggested that ions are accelerated at time of HXR maximum. This might also be the case of event 2 where the ion and (the relativistic) electron injection times roughly coincide. In the examined event, the strongest line broadening was seen near the reconnection site of the jet, however, at the time when the type III radio and HXR bursts terminated. This implies that the enhanced line broadening occurred at times when there was no energetic electron injection into interplanetary space or to the chromosphere. Unfortunately, this location was not scanned during the impulsive phase of the flare, though at the flare onset time no line broadenings were seen. Nevertheless, the observed excess in the line broadening appears not to be related to the wave turbulence that accelerated electrons (and presumably also the ions) otherwise energetic particle generation would continue.

Winglee (1989) has developed an acceleration mechanism related to the bulk flow evaporation, based on the ion-ion stream instability initiated by the precipitating energetic electrons. Though the observations in event 2 show a blueshift in the SEP source flare the strongest blueshift was observed in the weaker follow-up recurrent X-ray flare $(\sim 15$ minutes after the SEP event associated X-ray flare maximum) without a signature of particle acceleration. Reames et al. (1994) have doubted the mechanism as the events with chromospheric evaporation did not correlate with ${ }^{3} \mathrm{He}$-rich flares. The question whether such mechanism could operate in ${ }^{3} \mathrm{He}$-rich SEP flares remains still open. But it appears that the intensity of the blueshifted component alone may not be an appropriate indicator for the mechanism at work.

Diagnostic capabilities of EUV line spectroscopy for ion acceleration in solar flares need to be further explored, and possibly with better temporal resolution observations. Note that in the examined event, the area around the jet locus was not scanned during the critical impulsive phase that lasted $\sim 3-4$ minutes. Thus, we do not know when the excess broadening has started and how it evolved in time. For many flares, the excess broadening is observed to increase at the beginning of the impulsive phase (Fludra et al. 1989). Furthermore, near the flare onset when the background from the flaring chromosphere is small and charge-exchange is more efficient, as the medium is less ionized, the effect from the non-thermal ions may be better visible (Orrall \& Zirker 1976; Peter et al. 1990). More recent spectroscopic observations are performed with EIS and IRIS spectrometers. The EIS, in operation since November 2006, observes the solar corona at two wavelength bands, 170-210 and $250-290 \AA$ with $0.06 \AA$ spectral and $2^{\prime \prime}$ spatial resolution over a FOV of up to $360^{\prime \prime} \times 512^{\prime \prime}$ (Culhane et al. 2007). A preliminary check of the EIS flare catalog (http://solarb. mssl.ucl.ac.uk/SolarB/eisflarecat.jsp) indicates 10 out of 54 (January 2007-May 2014) already reported ${ }^{3} \mathrm{He}$-rich SEP source flares (Nitta et al. 2015; Bučík et al. 2016) in the EIS FOV. The IRIS provides UV spectra and images around $1400 \AA$ and $2800 \AA$ that focus on the chromosphere with $0.03 \AA$ spectral, $0.4^{\prime \prime}$ spatial, and 2-second temporal resolution over a FOV of up to $175^{\prime \prime} \times 175^{\prime \prime}$ (De Pontieu et al. 2014). So far only nine ${ }^{3} \mathrm{He}$-rich flares in the Earth view have been reported (Nitta et al. 2015) that cover an observing period of IRIS (since July 2013) but no one is found in the spectrograph FOV. An in- tentional pointing of the spectrometers to the candidate ${ }^{3} \mathrm{He}$-rich SEPs source (like an active region on near-equatorial coronal hole boundary on the western hemisphere) might provide more events for a systematic investigation.

Acknowledgements. This work was supported by the Deutsche Forschungsgemeinschaft (DFG) under grant BU 3115/2-1 and Max-Planck-Gesellschaft zur Förderung der Wissenschaften. ŠM acknowledges VEGA grant 2/0155/18.

\section{References}

Aptekar, R. L., Frederiks, D. D., Golenetskii, S. V., et al. 1995, Space Sci. Rev., 71,265

Bougeret, J.-L., Kaiser, M. L., Kellogg, P. J., et al. 1995, Space Sci. Rev., 71, 231 Brosius, J. W. 2001, ApJ, 555, 435

Brosius, J. W., \& Woodgate, B. E. 1999, ApJ, 514, 430

Bučík, R., Innes, D. E., Mall, U., et al. 2014, ApJ, 786, 71

Bučík, R., Innes, D. E., Mason, G. M., \& Wiedenbeck, M. E. 2016, ApJ, 833, 63 Bučík, R., Innes, D. E., Mason, G. M., et al. 2018, ApJ, 852, 76

Cane, H. V., Richardson, I. G., \& von Rosenvinge, T. T. 2010, J. Geophys. Res., 115, A08101

Canfield, R. C., \& Chang, C.-R. 1985, ApJ, 295, 275

Chen, N.-H., Bučík, R., Innes, D. E., \& Mason, G. M. 2015, A\&A, 580, A16

Culhane, J. L., Harra, L. K., James, A. M., et al. 2007, Sol. Phys., 243, 19

Czaykowska, A., De Pontieu, B., Alexander, D., \& Rank, G. 1999, ApJ, 521, L75

De Pontieu, B., Title, A. M., Lemen, J. R., et al. 2014, Sol. Phys., 289, 2733

Delaboudinière, J. P., Artzner, G. E., Brunaud, J., et al. 1995, Sol. Phys., 162, 291

Drake, J. F., Cassak, P. A., Shay, M. A., Swisdak, M., \& Quataert, E. 2009, ApJ, 700, L16

Fleishman, G. D., \& Toptygin, I. N. 2013, MNRAS, 429, 2515

Fludra, A. 2001, A\&A, 368, 639

Fludra, A., Lemen, J. R., Jakimiec, J., Bentley, R. D., \& Sylwester, J. 1989, ApJ, 344, 991

Fisk, L. A. 1978, ApJ, 224, 1048

Handy, B. N., Acton, L. W., Kankelborg, C. C., et al. 1999, Sol. Phys., 187, 229

Harrison, R. A., Sawyer, E. C., Carter, M. K., et al. 1995, Sol. Phys., 162, 233

Hudson, H. S., Fletcher, L., MacKinnon, A. L., \& Woods, T. N. 2012, ApJ, 752, 84

Jeffrey, N. L. S., Fletcher L., \& Labrosse, N. 2016, A\&A, 590, A99

Jeffrey, N. L. S., Fletcher L., \& Labrosse, N. 2017, ApJ, 836, 35

Kahler, S. W. 2007, Space Sci. Rev., 129, 359

Kahler, S. W., Reames, D. V., \& Sheeley Jr., N. R. 2001, ApJ, 562, 558

Kartavykh, Y. Y., Dröge, W., Klecker, B., et al. 2007, ApJ, 671, 947

Krucker, S., Larson, D. E., Lin, R. P., \& Thompson, B. J. 1999, ApJ, 519, 864

Krucker, S., Kontar, E. P., Christe, S., Glesener, L., \& Lin, R. P. 2011, ApJ, 742, 82

Kumar, R., Eichler, D., Gaspari, M., \& Spitkovski, A. 2017, ApJ, 835, 295

Laming, J. M., Moses, J. D., Ko, Y.-K., et al. 2013, ApJ, 770, 73

Leske, R. A., Wiedenbeck, M. E., Cohen, C. M. S., et al. 2003, in Proc. 28th ICRC (Tsukuba), 6, 3253

Lin, R. P., Anderson, K. A., Ashford, S., et al. 1995, Space Sci. Rev., 71, 125

Liu, S., Petrosian, V., \& Mason, G. M. 2006, ApJ, 636, 462

Mandzhavidze, N., Ramaty, R., \& Kozlovsky, B. 1999, ApJ, 518, 918

Mason, G. M. 2007, Space Sci. Rev., 130, 231

Mason, G. M., Wiedenbeck, M. E., Miller, J. A., et al. 2002, ApJ, 574, 1039

Mason, G. M., Mazur, J. E., Dwyer, J. R., et al. 2004, ApJ, 606, 555

McComas, D. J., Bame, S. J., Barker, P., et al. 1998, Space Sci. Rev., 86, 563

Messmer, P., Benz, A. O., \& Monstein, C. 1999, Sol. Phys., 187, 335

Miller, J. A. 1998, Space Sci. Rev., 86, 79

Milligan, R. O. 2011, ApJ, 740, 70

Milligan, R. O., Gallagher, P. T., Mathioudakis, M., \& Keenan, F. P. 2006a, ApJ, 642, L169

Milligan, R. O., Gallagher, P. T., Mathioudakis, M., et al. 2006b, ApJ, 638, L117 Moortel, I. De., \& Nakariakov, V. M. 2012, RSPTA, 370, 3193

Murphy, R. J., Ramaty, R., Kozlovsky, B., \& Reames, D. V. 1991, ApJ, 371, 793

Müller-Mellin, R., Kunow, H., Fleissner, V., et al. 1995, Sol. Phys., 162, 483

Nitta, N. V., Reames, D. V., DeRosa M. L., et al. 2006, ApJ, 650, 438

Nitta, N. V., Mason, G. M., Wang, L., Cohen, C. M. S., \& Wiedenbeck, M. E. 2015, ApJ, 806, 235

Orrall, F. Q., \& Zirker, J. B. 1976, ApJ, 208, 618

Peter, Th., Ragozin, E. N., Urnov, A. M., Uskov, D. B., \& Rust, D. M. 1990, ApJ, 351,317

Reames, D. V. 1988b, ApJ, 325, L53

Reames, D. V., \& Ng, C. K. 2004, ApJ, 610, 510 
Reames, D. V., \& Stone, R. G. 1986, ApJ, 308, 902

Reames, D. V., von Rosenvinge, T. T., \& Lin, R. P. 1985, ApJ, 292, 716

Reames, D. V., Dennis, B. R., Stone, R. G., \& Lin, R. P. 1988a, ApJ, 327, 998

Reames, D. V., Meyer, J. P., \& von Rosenvinge, T. T. 1994, ApJS, 90, 649

Reames, D. V., Cliver, E. W., \& Kahler, S. W. 2014, Sol. Phys., 289, 3817

Scherrer, P. H., Bogart, R. S., Bush, R. I., et al. 1995, Sol. Phys., 162, 129

Shibata, K., Ishido, Y., Acton, L. W., et al. 1992, PASJ, 44, L173

Smith, C. W., L'Heureux, J., Ness, N. F., et al. 1998, Space Sci. Rev., 86, 613

Tan, C., \& Yan, Y. 2008, AdSpR, 41, 992

Temerin, M., \& Roth, I. 1992, ApJ, 391, L105

Teriaca, L., Falchi, A., Falciani, R., Cauzzi, G., \& Maltagliati, L. 2006, A\&A, 455,1123

Van Hollebeke, M. A. I., McDonald, F. B., \& Meyer, J. P. 1990, ApJS, 73, 285

Vilmer, N., MacKinnon, A. L., \& Hurford, G. J. 2011, Space Sci. Rev., 159, 167

Wang, Y.-M., Pick, M., \& Mason, G. M. 2006, ApJ, 639, 495

Wang, L., Lin, R. P., Krucker, S., \& Mason, G. M. 2012, ApJ, 759, 69

Wiedenbeck, M. E., Cohen, C. M. S., Leske, R. A., et al. 2010, ApJ, 719, 1212

Winglee, R. M. 1989, ApJ, 343, 511

Woodgate, B. E., Robinson, R. D., Carpenter, K. G., Maran, S. P., \& Shore, S. N. 1992, ApJ, 397, L95

Zhang, T. X. 2004, ApJ, 617, L77 